\title{
Sur deux Cimex (Insecta, Heteroptera), nouveaux pour la faune française, parasites des chauves-souris
}

\author{
par R.-L. USINGER et J.-C. BEAUCOURNU
}

L'un de nous (Beaucournu, 1961) avait antérieurement signalé la présence dans l'Ouest de la France d'un Cimex parasitant les chauves-souris dans deux colonies de mise bas ; leur identité précise n'avait pu alors être affirmée. Nous avons pu réétudier de nombreux exemplaires de ces deux stations et en avons découvert une troisième. Ces captures, représentant une forme et une espèce nouvelles pour la France, font l'objet de cette note (1).

Nous devons rappeler que quatre espèces de Cimicidés furent, jusqu'à présent, signalées de France : $C$. lectularius Merrett, $C$. pipistrelli Jenyns, $C$. columbarius J. et Oecacius hirundinis J. Sur ces quatre punaises, une, $C$. pipistrelli, ne semble pas avoir été effectivement capturée dans ce pays; une autre, $C$. columbarius, longtemps dotée d'un statut taxonomique douteux, est définitivement considérée comme une espèce distincte. Elle n'a pas été avec certitude identifiée de France ces dernières années, et d'ailleurs, toutes les références la concernant ne s'appuyant pas sur une étude chromosomique doivent être tenues pour douteuses ou erronées.

\section{CIMEX LECTULARIUS MERRETT 1667}

Tous les $C$. lectularius européens étudiés jusqu'à présent (Usinger, 1966) étaient caractérisés par un équipement chromosomique comportant des chromosomes surnuméraires (13 autosomes $+6 \mathrm{X}+6 \mathrm{Y})$. Ils se différenciaient de tous les $C$. lectularius américains connus qui sont 13 autosomes $+2 \mathrm{X}+\mathrm{Y}$ (nombre haploïde, $2^{\circ}$ métaphase).

Les Cimex prélevés dans deux colonies de chauves-souris du Maine-et-Loire (Durtal et Blou) appartiennent au type américain : ce sont les deux seuls gîtes connus en Europe de cette forme.

(1) Nous remercions bien vivement le $D^{r} N$. Ueshima responsable du travail cytologique dans l'identification de ce matériel. 
La densité de cette espèce est très élevée, particulièrement à Durtal, et nous rappelons ce que l'un de nous avait écrit à ce propos : « Elle habite les fissures des poutres en colonies impressionnantes. La récolte d'une population groupée sur deux ou trois décimètres carrés, en hibernation, c'est-à-dire à un moment où l'espèce est classiquement moins abondante, et en négligeant les spécimens installés dans les fentes trop étroites pour nos pinces, nous a fourni 47 mâles, 33 femelles et 27 larves. Nous ne donnons cet exemple que parce qu'il est chiffré, mais nous sommes persuadés que ce total n'a rien d'exceptionnel. »

Il est à souligner que, si la colonie de Blou vit dans les combles d'une église isolée sur une place, où par conséquent les punaises sont obligatoirement liées aux chauvessouris hôtes (celles-ci étant absentes pendant tout l'hivernage), par contre, à Durtal, ces Insectes vivent dans le grenier du château où ils se gorgent aux dépens d'une colonie de mise bas de Myotis myotis (Borkhausen). Celle-ci n'est constituée que du 15 avril au 15 septembre approximativement. Il serait logique de penser que, pendant l'absence de leurs hôtes, les punaises quittent le grenier et descendent dans les pièces immédiatement inférieures où est installé un hospice de vieillards. Or, il semble n'en être rien et les Cimex paraissent hiberner eux aussi et sont trouvés, à cette époque, immobiles dans les fissures de poutres de leur gîte estival. Un tel comportement est aberrant pour Cimex lectularius.

Rappelons que les chauves-souris hôtes sont essentiellement, à Durtal, Myotis myotis, à Blou, Rhinolophus ferrumequinum (Schreber), $R$. euryale Blasius et $M$. emarginatus (Geoffroy).

\section{CIMEX STADLERI HORVATH 1935}

Cette rare espèce inféodée aux chauves-souris fut décrite d'Allemagne, de la région du Spessart, soit à $180 \mathrm{~km}$ au N.-E. de Strasbourg. Horväth signala qu'elle fut prélevée dans une colonie de Vespertilio murinus. Sous ce nom, on a désigné tantôt Myotis myotis, tantôt $V$. discolor. Il doit logiquement s'agir de la première de ces deux chauvessouris, beaucoup plus fréquente que la seconde dans cette région et particulièrement dans le gîte mentionné ici : le grenier d'une école. A notre connaissance, cette punaise ne fut jamais signalée en France.

Nous en avons récolté cinq exemplaires ( 2 ô, 2 \&, 1 1.), le 20 juin 1964, sous une planche reposant sur du guano de chauves-souris dans les combles de l'église Notre-Dame-des-Marais, à la Ferté-Bernard (Sarthe). Elle vit dans cette colonie aux dépens de deux espèces: Rhinolophus ferrumequinum et Myotis emarginatus, mais, comme pour les autres colonies signalées ici, ce gîte est déserté l'hiver par les chiroptères. Il faut toutefois mentionner la possibilité, théorique, qu'ont ces punaises de se nourrir sur d'autres animaux: en effet, les anfractuosités des murailles de cette église abritent de nombreux choucas (Coloeus monedula L.). 


\section{Bibliographie}

Beaucournu (J.-C.), 1961. - Ectoparasites des Chiroptères de l'Ouest de la France. $1^{\text {re }}$ partie : Ixodoïdés, Cimicidés et Nyctéribiidés. Bull. Soc. Sc. Bretagne, 36, 315.

Usinger (R.-L.), 1966. - Monograph of Cimicidae. The Thomas Say Foundation, Entomological Society of America, Vol. VII., sous presse.

(Division of Entomology and Acaralogy, College of Agriculture, Berkeley, California et Laboratoire de Parasitologie et Zoologie appliquée de la Faculté de Médecine de 35 - Rennes) 